Pacific Journal of Mathematic 


\section{NOTES ON IDEAL COVERS AND ASSOCIATED PRIMES}

\section{J. RATliff, JR. AND DAvid E. Rush}

The covering relationship between ideals $(B \subset C$ and there exist no ideals between $B$ and $C$ ) is studied, and, surprisingly, due to an interesting interplay between the concepts, quite a few new results concerning Bourbaki associated primes are found. Then, most of the results are generalized to submodules of an an arbitrary $A$-module.

The covering relationship between ideals has proved to be a useful and important tool in many investigations in commutative algebra. For example, a result in a classical paper of Gröbner [2, $\S 6]$ says that an $M$-primary ideal $Q$ in a local $\operatorname{ring}(A, M)$ is irreducible if and only if $Q$ has a unique cover. (See also [9, p. 248].) (Other examples include most papers where one of the following concepts is considered: the length of an ideal, a minimal basis of an ideal, or the Hilbert-Samuel polynomial of an ideal.) However, the authors know of no paper where the subject itself has been studied. We do such a study in $\S 2$ of this paper, and some quite interesting results are obtained. Also, it turns out that there is an interesting interplay between the covering relationship and Bourbaki associated primes, so results concerning one of the concepts imply corresponding results concerning the other concept. Therefore in $\S 3$, using the results of $\S 2$, quite a few new results concerning Bourbaki associated primes of an ideal in an arbitrary ring are obtained. (In particular, all the results hold for prime divisors of an ideal in a Noetherian ring, and many are new even in this case.) That many new results are obtained is somewhat surprising, since the subject of Bourbaki associated primes has certainly previously been deeply investigated.

To briefly describe the results in $\S 2$, let $B \subset C$ be ideals in a ring $A$. Then we say that $C$ covers $B$ in case there are no ideals $D$ of $A$ such that $B \subset D \subset C$. In this case, $C / B \cong A / M$, for some maximal ideal $M$, and to emphasize the role of $M$ we say that $C M$ covers $B$. With this terminology, it is first shown in $\S 2$ that an ideal $I$ in $A M$-covers some ideal if and only if $M I \neq I(2.3)$, so if $A$ is either quasi-local or an integral domain, then every nonzero finitely generated ideal $M$-covers some ideal (2.4). Some other corollaries of (2.3) are given in (2.5)-(2.10), among which is a description of an ideal which $M$-covers a unique ideal - a result which is sort of dual to the above mentioned result of Gröbner. Next it is 
shown that if an ideal $C M$-covers $B$ and $I$ is any ideal in $A$, then either $C \cap I M$-covers $B \cap I$ or $C+I M$-covers $B+I$ (2.13). Consequences of this are given in (2.14)-(2.16), among which are: if $C+I$ $M$-covers $B+I$, then $C+J M$-covers $B+J$, for all ideals $J \subseteq I$ (2.14.1); if $d$ is a regular element in $A$, then either $C: d A=B: d A$ or $C: d A M$-covers $B: d A$ (2.14.4); and, if $d I$ is $M$-covered ( $d$ regular), then either $d A$ or $I$ is $M$-covered (2.16). (2.17) gives a form of a converse of part of (2.13) for Noetherian rings, and $\S 2$ is closed by showing, in particular, that if $C$ covers $B$ in a local ring $R$, then, for all ideals $I$ in $R, C+I^{e}$ covers $B+I^{e}$, for all large $e(2.20)$.

Section 3 is concerned with Bourbaki associated prime ideals $P$ of an ideal $I(P=I: x A$, for some $x \in A)$. The first result shows that if $M$ is maximal, then $M$ is a Bourbaki associated prime of $I$ if and only if $I$ is $M$-covered (3.2). Using this, a number of additional corollaries of (2.3) are given, among which are: if $M I \neq I$, then $M I$ is an intersection of ideals lying immediately below $I$ and having $M$ as a Bourbaki associated prime (3.3); and, every ideal in a ring is an intersection of ideals having a maximal ideal as a Bourbaki associated prime (3.5). In (3.10) it is shown that a finitely generated prime ideal $P$ in a ring $A$ is a Bourbaki associated prime ideal of an ideal $B$ if and only if $B A_{P}$ is a covered ideal. Corollaries of this which correspond to results in $\S 2$ are given in (3.11)-(3.14), among which are: if $P$ is a Bourbaki associated prime of $B$ and is either finitely generated or a maximal ideal in $A$, then, for each ideal $I$ in $A, P$ is a Bourbaki associated prime of either $B+J$ (for all ideals $J \subseteq I$ ) or $B \cap K$ (for all ideals $K \supseteqq I$ ) (3.11); moreover, if $P$ is not a Bourbaki associated prime of $B+d A$ ( $d$ regular), then $P$ is a Bourbaki associated prime of $B: d A$ (3.12.3); and, if $P$ is a Bourbaki associated prime of $d I$ ( $d$ a regular element), then $P$ is a Bourbaki associated prime of either $d A$ or $I$ (3.13). In (3.15) it is shown that if $P$ is a prime divisor of an ideal $B$ in a Noetherian ring $A$, then, for all ideals $I \subseteq P, P$ is a prime divisor of $B+I^{e}$, for all large $e$. Corollaries of this are given in (3.15)-(3.19), among which is: if $P$ is a prime divisor of (0), then, for all ideals $I \cong P, P$ is a prime divisor of $I^{e}$, for all large $e$ (3.16.1).

In $\S \S 4$ and 5 , we briefly indicate how the results in $\S \S 2$ and 3 can be generalized to, respectively, the module-covering relationship between submodules of an arbitrary $A$-module $\mathscr{l}$, and to Bourbaki associated primes of submodules of $\mathscr{K}$. It turns out that most of the results in $\S \S 2$ and 3 can be generalized to this case. (The main reason for doing the ring and module cases separately is that the notation and arguments are a little simpler in the ring case, and once this case is known, the module case follows quite readily. A secondary reason is that a few of the results for the ring case 
do not have a natural generalization to modules.) It should be mentioned that results analogous to those in this paper have been obtained for Noetherian lattice modules in [4].

Finally, in $\S 6$ a few remarks are made concerning the more restrictive condition (than being covered) of a sheltered submodule of an $A$-module.

2. Notes on ideal covers. All rings in this article are assumed to be commutative with an identity. The undefined terminology is, in general, the same as that in [5]. In particular, $B \subset C$ means the set $B$ is properly contained in the set $C$. Also, if $B$ is an ideal in a ring $A$, then possibly $B=A$, but if $B$ is a prime ideal in $A$, then $B \subset A$.

In this section we prove a number of results concerning the covering relationship between ideals in a ring. We begin by recalling the following definition.

Definition 2.1. Let $B, C$, and $M$ be ideals in a ring $A$. Then it is said that $C M$-covers $B$ if $B \subset C, M$ is maximal, and $C / B \cong A / M$ (as vector spaces). It is said that $C$ covers $B$, if $C N$-covers $B$ for some maximal ideal $N$ in $A$, and that $B$ is covered, if some ideal covers $B$.

The following remark lists a few immediate equivalences of the definitions.

REMARK 2.2. Let $B \subset C$, and $M$ be ideals in a ring $A$ such that $M$ is maximal. Then the following statements hold:

(2.2.1) $C$ covers $B$ if and only if $C / B$ is a nonzero simple $A$ module, or, equivalently, if and only if there does not exist an ideal $I$ in $A$ such that $B \subset I \subset C$.

(2.2.2) [9, Corollary 1, p. 237]. $C M$-covers $B$ if and only if $M C \cong B$ and $C=B+c A$, for some $c \in C, \notin B$.

(2.2.3) $C M$-covers $B$ if and only if $C=B+c A$ and $M=B: C=$ $B: c A$, for some (and then for every) $c \in C, \notin B$.

The next result and its corollaries show the ubiquity of the ideal covering relationship.

Theorem 2.3. Let $I$ and $M$ be ideals in a ring $A$ such that $M$ is maximal. Then $I$ M-covers some ideal $J$ in $A$ if and only if $M I \neq I . \quad$ If $M I \neq I$, then the intersection of the ideals $J$ such that $I M$-covers $J$ is $M I$.

Proof. If $I M$-covers some ideal $J$, then $M I \subseteq J \subset I$ (2.2.2), so $M I \neq I$. 
If $M I \neq I$, then let $x \in I, \notin M I$. Extend $\{x+M I\}$ to a basis $\beta$ of the $A / M$-vector space $I / M I$, and let $V$ be the subspace of $I / M I$ generated by $\beta-\{x+M I\}$. Let $\sigma$ be the canonical map of $I$ onto $I / M I$. Then $J=\sigma^{-1}(V)$ is $M$-covered by $I$ and $x \notin J$, so $M I$ contains the intersection of the ideals $M$-covered by $I$. On the other hand, if $I M$-covers $J$, then $M I \subseteq J(2.2 .2)$, so $M I$ is the intersection of the ideals $M$-covered by $I$.

(We are indebted to the referee for (2.4) and (2.6), both of which sharpen our original results.)

Corollary 2.4. Let $I \neq(0)$ and $M$ be ideals in a ring $A$ such that $I$ is finitely generated and $M$ is maximal. Then there exists an ideal $J$ in $A$ such that $I M$-covers $J$ if and only if $(0): I \subseteq M$. (Thus, if $A$ is either quasi-local or an integral domain, then $I M$ covers some ideal $J$.)

Proof. If $I M$-covers $J$, then (0): $I \subseteq J: I=M$, by (2.2.3). Conversely, assume that $(0): I \subseteq M$ and suppose that $M I=I$. Then, by [3, Theorem 76], there exists $x \in M$ such that $(1+x) I=(0)$, so $1+x \in(0): I \subseteq M$. But this is a contradiction, since $x \in M$, so $M I \neq I$, and so the conclusion follows from (2.3). The parenthetical statement follows immediately from this.

REMARK 2.5. Let $I \neq(0)$ and $M$ be ideals in a ring $A$ such that $I$ is finitely generated and $M$ is maximal. Then it follows from (2.4) and (2.3) that $I M$-covers some ideal in $A$ if and only if (0): $I \cong M$ if and only if $M I \neq I$, and then $M I=\cap\{J ; J$ is an ideal in $A$ and $I M$-covers $J\}$.

(2.6) is related to [1, Chapter 3, Ex. 18a, p. 110], which is concerned with sheltered modules. So as not to delay developing properties of the ideal covering relationship, we simply note here that the first statement in (2.6) is sharpened by the referenced result in [1]. (See (6.2.2) - and see $\S 6$ for additional results on sheltered modules.)

Corollary 2.6. For each ring $A$ and for each ideal $D \neq A$, $D$ is an intersection of covered ideals. Further, every nonzero ideal $I$ contains a covered ideal.

Proof. Let $x \in A, \notin D$, and let $M$ be a maximal ideal in $A$ such that $D: x A \subseteq M$. Then $x \notin D+x M$, for otherwise $x=d+x m$, for some $d \in D$ and $m \in M$, so $x(1-m)=d$, hence $1-m \in D: x A \subseteq M$, and this is a contradiction. Now expand $D+x M$ to an ideal $B$ 
maximal with respect to not containing $x$, and let $C=B+x A$. Then $C$ covers $B$ (by (2.2.1), since there exist no ideals between $C$ and $B$, by the definition of $B$ ), and $x \notin B$. Therefore, since $D \cong B$, it follows that $D$ is the intersection of covered ideals.

If $I$ is a nonzero ideal in $A$, then $I$ contains a nonzero finitely generated ideal in $A$, so the last statement follows from (2.4).

In (2.7) we consider when an ideal is an intersection of $M$-covered ideals, with $M$ a fixed maximal ideal.

Corollary 2.7. Let $I \cong M$ be ideals in a ring $A$ such that $M$ is maximal. Then $I$ is an intersection of $M$-covered ideals in the following two cases:

(2.7.1) I is prime.

(2.7.2) $M$ is finitely generated and $I=I A_{M} \cap A$.

Proof. (2.7.1) By passing to $A / I$, it may be assumed that $I=(0)$, and it may clearly be assumed that $I \neq M$ (since $A M$-covers $M)$. Then, for each nonzero element $a \in M, a M$ is $M$-covered by $a A$ $(\alpha M \subset a A$, since $A$ is a domain), and $(0)=\cap\{a A ; 0 \neq a \in M\} \supseteqq$ $\cap\{a M ; 0 \neq a \in M\} \supseteqq(0)$, so (2.7.1) holds.

(2.7.2) By (2.6), $I A_{M}$ is an intersection of $M A_{M}$-covered ideals $B A_{M}$, so $I A_{M} \cap A$ is an intersection of the ideals $B A_{M I} \cap A$, and each of these ideals is $M$-covered, by (3.10) and (3.2).

Corollary 2.8. Let $M, I$, and $A$ be as in (2.3). Then $I M$ covers a unique ideal $J$ in $A$ if and only if $I=M I+x A$, for some $x \in I$, $\notin M I$, and then $J=M I$.

Proof. If $I M$-covers $J$, then there exists $x \in I, \notin J$ such that $I=J+x A$ and $x M \subseteq J$ (2.2.2). If $J$ is unique, then $J=M I$ (2.3), so $I=M I+x A$ and $x \in I, \notin M I$.

Conversely, if there exists $x \in I, \notin M I$ such that $I=M I+x A$, then $I M$-covers $M I(2.2 .2)$. If also $I M$-covers $K$, then $M I \subseteq K$ (2.2.2), so necessarily $K=M I$.

Corollary 2.9. Let $M$, $I$, and $A$ be as in (2.3) and assume that $I M$-covers a unique ideal. Then there exists $x \in I$ such that $I=\cap\left\{x A+M^{i} I ; i \geqq 1\right\}$. Therefore, if $A$ is quasi-local and $I$ is finitely generated, then $I$ is principal.

Proof. By (2.8), there exists $x \in I$ such that $I=x A+M I$, so $M I=x M+M^{2} I$, hence $I=x A+M^{2} I$. Therefore it follows that 
$I=\cap\left\{x A+M^{i} I ; i \geqq 1\right\}$. Therefore, if $A$ is quasi-local and $I$ is finitely generated, then, since $I=x A+M I,[5,(5.1)]$ says that $I=x A$.

REMARK 2.10. Let $M, I$, and $A$ be as in (2.3), and assume that $(0) \neq I=x A$ is a principal ideal and $A$ is either a domain or a quasilocal ring. Then $I M$-covers a unique ideal (namely, $M I$ ). (Therefore, for a finitely generated ideal $I$ in a quasi-local ring $(A, M), I$ $M$-covers a unique ideal if and only if $I$ is principal.)

Proof. Since $A$ is either a domain or a quasi-local ring and $I=x A, x M \neq x A$. Thus, by (2.2.2), $x A M$-covers $x M$. If also $x A$ $M$-covers $J$, then $x M \subseteq J(2.2 .2)$, so $J=x M$. The parenthetical statement follows from this and (2.9).

A number of other corollaries of (2.3) concerning Bourbaki associated primes could now be given. However, we postpone giving them till $\S 3$, since we want to concentrate on the ideal covering relationship in this section. The corollaries are given in (3.3)-(3.6).

The next result gives some further information on the $M$-covering relationship. Whereas (2.3) gave a necessary and sufficient condition for a given ideal to $M$-cover some ideal, (2.11) gives a necessary and sufficient condition for ideals of the form $D+x A$ to $M$-cover some ideal $B \supseteqq D$.

Proposition 2.11. Let $D$ and $M$ be ideals in a ring $A$ such that $M$ is maximal, and let $x \in A$. Then $D+x A M$-covers some ideal $B \supseteqq D$ if and only if $D: x A \subseteq M$. If $D+x A M$-covers $B \supseteqq D$, then $B=D+x M$.

Proof. The kernel of the natural homomorphism of $A$ onto $(D+x A) / D$ is easily seen to be $D: x A$, so $A /(D: x A) \cong(D+x A) / D$. Therefore the maximal submodules of $(D+x A) / D$ are in one-to-one correspondence to the maximal ideals containing $D: x A$. For the last statement, define $\sigma: A /(D: x A) \rightarrow(D+x A) / D$ by $\sigma(a+(D: x A))=$ $a x+D$. Then $\sigma(M+(D: x A))=D+x M$.

(An alternate proof of (2.11) can be given using (2.4) and (2.2.2).)

REMARK 2.12. Let $D, M$, and $A$ be as in (2.11). Then the following statements hold:

(2.12.1) If $D \subseteq M$, then, for all $x \in A, \notin M, D+x A M$-covers $D+x M$.

(2.12.2) If $A$ is quasi-local and $D$ is $M$-covered, then $D+I M$ is $M$-covered, for all finitely generated ideals $I$ in $A$. 
Proof. (2.12.1) If $D \subseteq M$ and $x \notin M$, then $D: x A \subseteq M: x A=M$, so the conclusion follows from (2.11).

(2.12.2) If $x \in I \cap D$, then $D+x M=D$ is covered, by hypothesis; and if $x \in I, \notin D$, then $D: x A \subseteq M$, so $D+x M$ is $M$-covered, by (2.11). Since $I$ is finitely generated, the conclusion follows by a finite number of repetitions of this.

(3.7) and (3.8) are additional corollaries of (2.11) concerning Bourbaki associated primes.

The following result shows an interesting property of ideal covers.

Theorem 2.13. Let $B, C, I$, and $M$ be ideals in a ring $A$ such that $M$ is maximal and $C M$-covers $B$, and consider the following statements:

(2.13.1) $\quad C \cap I \nsubseteq B$.

(2.13.2) $B \cap I \subset C \cap I$.

(2.13.3) $\quad(B \cap I):(C \cap I)=B: C$.

(2.13.4) $C \cap I$-covers $B \cap I$.

(2.13.1') $\quad C \nsubseteq B+I$.

$\left(2.13 .2^{\prime}\right) \quad B+I \subset C+I$.

(2.13.3') $(B+I):(C+I)=B: C$.

(2.13.4') $C+I M$-covers $B+I$.

Then (2.13.1)-(2.13.4) are equivalent and (2.13.1')-(2.13.4') are equivalent. Moreover, exactly one set of the statements (2.13.1)(2.13.4) or $\left(2.13 .1^{\prime}\right)-\left(2.13 .4^{\prime}\right)$ holds.

Proof. $C \cap I \subseteq B$ if and only if $C \cap I \subseteq B \cap I$ if and only if (by hypothesis) $C \cap I=B \cap I$, so (2.13.1) $\Leftrightarrow(2.13 .2)$.

If $C \cap I \nsubseteq B$, then $B+(I \cap C)=C$ (since $C$ covers $B)$, so $A / M \cong$ $C / B=(B+(I \cap C)) / B \cong(I \cap C) /(B \cap(I \cap C))=(I \cap C) /(I \cap B)$, and so $C \cap I M$-covers $B \cap I$ (2.1), hence $(2.13 .1) \Rightarrow(2.13 .4)$.

Assume that (2.13.4) holds. Then, by (2.2.3), $(B \cap I):(C \cap I)=$ $M=B: C$, hence $(2.13 .4) \Rightarrow(2.13 .3)$.

It is clear that $(2.13 .3) \Rightarrow(2.13 .2)$.

$C \leqq B+I$ if and only if $C+I \subseteq B+I$ if and only if (by hypothesis) $C+I=B+I$, so $\left(2.13 .1^{\prime}\right) \Leftrightarrow\left(2.13 .2^{\prime}\right)$.

If $B+I \subset C+I$, then, with $C=B+c A$ (2.2.2), $C+I=B+I+c A$ and $M(B+I+c A) \subseteq B+I$, hence $C+I M$-covers $B+I$ (2.2.2). Therefore $\left(2.13 .2^{\prime}\right) \Rightarrow\left(2.13 .4^{\prime}\right)$.

Assume that (2.13.4') holds. Then, by (2.2.3), $(B+I):(C+I)=$ $M=B: C$, hence $\left(2.13 .4^{\prime}\right) \Rightarrow\left(2.13 .3^{\prime}\right)$.

It is clear that $\left(2.13 .3^{\prime}\right) \Rightarrow\left(2.13 .2^{\prime}\right)$.

Finally, let $J=B+(C \cap I)$, so, by modularity, $J=, C \cap(B+I)$. 
Also $B \subseteq J \subseteq C$. Therefore, either $J=B$ or $J=C$. Now $J=B$ if and only if $C \cap I \subseteq B$, and $J=C$ if and only if $C \subseteq B+I$. Therefore, if $J \neq B$, then $C \cap I \nsubseteq B$, so $C \cap I M$-covers $B \cap I$ (by what has already been shown); and, if $J \neq C$, then $C \nsubseteq B+I$, so $C+I$ $M$-covers $B+I$ (by what has already been shown). Also, $J=B$ if and only if $C \cap I \subseteq B$ if and only if $C \cap I$ does not $M$-cover $B \cap I$, and $J=C$ if and only if $C \subseteq B+I$ if and only if $C+I$ does not $M$-cover $B+I$, so exactly one of (2.13.4) and (2.13.4') holds.

The statements in the following remark follow immediately from (2.13).

REMARK 2.14. With the notation of (2.13), let $c \in C, \notin B$, so $C=$ $B+c A(2.2 .3)$. Then the following statements hold:

(2.14.1) $C+I M$-covers $B+I$ if and only if $c \notin B+I$. Therefore, if $C+I M$-covers $B+I$, then, for all ideals $J \subseteq I, C+J M$ covers $B+J$. Also, there exist ideals $I$ which are maximal with respect to the property that $C+I M$-covers $B+I$.

(2.14.2) If $C \cap I M$-covers $B \cap I$, then $C \cap J M$-covers $B \cap J$, for all ideals $J \supseteqq I$.

(2.14.3) Either $C \cap I=B \cap I$ or $C \cap I M$-covers $B \cap I$; and, either $C+I=B+I$ or $C+I M$-covers $B+I$.

(2.14.4) For each regular element $d$ in $A$, either $C: d A=B: d A$ or $C: d A M$-covers $B: d A$, since $C \cap d A=d(C: d A)$.

(2.14.5) If $I+B \nsubseteq M=B$ : $C$, then $C \cap I M$-covers $B \cap I$.

(2.14.6) A $M$-covers $M$, so, for all ideals $I \nsubseteq M, I M$-covers $M \cap I$ (so, for each $a \in I, \notin M, I=(M \cap I)+a A$ ).

It follows from (2.13.4) that if $C_{i} M$-covers $B_{i}(i=1,2)$, then either: $C=C_{1} \cap C_{2}=B_{1} \cap B_{2}=B$; or $C$ covers $B$; or $C$ covers $C_{1} \cap B_{2}$ covers $B$ and $C$ covers $C_{2} \cap B_{1}$ covers $B$. Thus, it probably is not true that if $C M$-covers $B$, then either $C: I=B: I$ or $C: I M$-covers $B: I$, for all ideals $I$ in $A$. However, see (2.18.1).

Two corollaries to (2.13) will now be given. The first is a special case of parts of (2.14), but it is felt that it is of sufficient importance to be specifically mentioned.

Corollary 2.15. Let $B, C, I$, and $M$ be ideals in a ring $A$ such that $M$ is maximal and $C M$-covers $B$. Then the following statements hold:

(2.15.1) If $C \cap I^{e}=B \cap I^{e}$, for some $e \geqq 1$, then $C+I^{i} M$-covers $B+I^{i}$, for all $i \geqq e$.

(2.15.2) If $C+I^{e}=B+I^{e}$, for some $e \geqq 1$, then $C \cap I^{i} M$-covers $B \cap I^{i}$, for $i=1, \cdots, e$.

(2.15.3) If $C+d^{e} A=B+d^{e} A$, for some $e \geqq 1$, and if $d$ is 
regular, then $C: d^{i} A M$-covers $B: d^{i} A$, for $i=1, \cdots, e$.

Of course, the contrapositive of (2.15.3) shows that if $C: d^{e} A=$ $B: d^{e} A$, for some $e \geqq 1$, then $C+d^{i} A M$-covers $B+d^{i} A$, for all $i \geqq e$.

Corollary 2.16. Let $I$ and $M$ be ideals in a ring $A$ such that $M$ is maximal, and let $d$ be a regular element in $A$. If $d I$ is $M$ covered, then either $d A$ or $I$ is $M$-covered.

Proof. Let $C M$-cover $d I$. If $d A$ is not $M$-covered, then $C+d A=$ $d I+d A=d A$ (2.14.3), so $I=d I: d A$ is $M$-covered (2.15.3).

(2.16) shows that if $d^{e} I$ is $M$-covered, for some $e \geqq 1$, then either $d A$ is $M$-covered or $d^{i} I$ is $M$-covered, for $i=0,1, \cdots, e$. Namely, (2.1) shows that if $d A$ is not $M$-covered, then $d^{e-1} I$ is $M$-covered (since $d^{e} I=d\left(d^{e-1} I\right)$ ), so the conclusion follows by repetition.

If $A$ is Noetherian and $Q$ is an $M$-primary ideal of $A$, for some maximal ideal $M$, then, since $A / Q$ is Artinian, it is clear that $Q$ is $M$-covered. This together with (2.13) gives a large class of covered ideals. The next result shows that, in fact, every covered ideal in $A$ can be obtained in this way, in that, if $C M$-covers $B$, then the primary decompositions of $B$ and $C$ differ only in $M$-primary components and some $M$-primary component of $B$ is either covered by some $M$-primary component of $C$ or $C$ has no $M$-primary component. Also, (2.17) shows that by considering a primary decomposition of a covered ideal $B$, we can build up from $B$ to an ideal which covers it, instead of the other way around as in (2.3)-(2.5) and (2.8)-(2.11).

Before stating (2.17), we give an example to show that $C$ may not have an $M$-primary component even when $M$ is not the component of $B$.

ExAmple. Let $(R, M=(a, b))$ be a regular local ring, let $Q=$ $\left(a, b^{3}\right) R$ (so $\left.\left(a, b^{3}\right) R \subset\left(a, b^{2}\right) R \subset M\right)$, let $x=a^{2}+b^{2}$, let $B=Q \cap x R$, and let $C=x R$. Then $C M$-covers $B$ (since $B=Q \cap x R=x(Q: x R)=$ $x M$, and $x R M$-covers $x M$, by (2.10)) and $C$ has no $M$-primary component (since $C$ is a principal ideal and $R$ is a Krull domain).

Proposition 2.17. Let $B \subset C$ and $M$ be ideals in a Noetherian ring $A$ such that $M$ is maximal and $C M$-covers $B$. Then $B$ has an $M$-primary component, $B$ and $C$ have the same primary decompositions except for $M$-primary components, and either some $M$ primary component of $C M$-covers some $M$-primary component of $B$ or $C$ has no $M$-primary component. 
Proof. If $B=M$, then $C=A$ and the conclusion is clear. If $B$ is $M$-primary and $B \neq M$, then $C$ is $M$-primary and the conclusion is again clear. Therefore assume that $B$ is not $M$-primary.

Now $M=B: C$, since $C M$-covers $B$, so $M$ is a prime divisor of $B$. Also, for each prime ideal $P \neq M$ in $A, C A_{P}=M C A_{P} \subseteq B A_{P} \subseteq$ $C A_{P}$, hence it follows that there exist primary ideals $q, Q_{2}, \cdots, Q_{n}$ in $A$ such that $q$ is $M$-primary, $q \cap Q_{2} \cap \cdots \cap Q_{n}$ is a normal primary decomposition of $B$, and either $Q_{2} \cap \cdots \cap Q_{n}$ is a normary decomposition of $C$ or $q^{\prime} \cap Q_{2} \cap \cdots \cap Q_{n}$ is a normal primary decomposition of $C$, for some $M$-primary ideal $q^{\prime}$. Therefore it remains to show that if $M$ is a prime divisor of $C$, then $q$ and $q^{\prime}$ can be chosen such that $q^{\prime} M$-covers $q$. For this, it may be assumed that $q \subseteq q^{\prime}$, since $B \subset C$, so $q \subset q^{\prime}$. Therefore let $I=Q_{2} \cap \cdots \cap Q_{n}$, let $q=q_{1} \subset \cdots \subset q_{k}=q^{\prime}$ be a chain of $M$-primary ideals such that $q_{i}$ covers $q_{i-1}$, for $i=2, \cdots, k$, and let $B_{i}=I \cap q_{i}(i=1, \cdots, k)$. Then $B=B_{1} \subseteq \cdots \subseteq B_{k}=C$, so, since $C$ covers $B$, there exists $d<k$ such that $B=B_{1}=\cdots=B_{d} \subset$ $B_{d+1}=\cdots=B_{k}=C$, and the conclusion follows immediately from this.

(2.17) holds in the more general case that every ideal in $A$ is a finite intersection of primary ideals and, for all maximal ideals $M$ and $M$-primary ideals $Q, A / Q$ is Artinian (by the proof of (2.17)).

This section will be closed with three more results concerning ideal covers in a Noetherian ring.

REMARK 2.18. Let $C M$-cover $B$ in a Noetherian ring $A$, and let $J$ be an ideal in $A$. Then the following statements hold:

(2.18.1) If $J \subseteq B: C$, then $C: J^{i}=B: J^{i}$, for all large $i$.

(2.18.2) Let $Q$ be an $M$-primary component of $B$ (2.17). If $J \subseteq Q$, then $C+J^{i} M$-covers $B+J^{i}$, for all $i \geqq 1$.

Proof. (2.18.1) If $J \subseteq B: C$, then (2.18.1) follows immediately from (2.17), since, for all large $i, J^{i}$ is contained in a given $M$-primary component of $B$.

(2.18.2) If $J \subseteq Q$, then $C \cap J=B \cap J$ (2.17), so the conclusion follows from (2.15.1).

REMARK 2.19. With the notation of (2.18.2), if $I_{1}, \cdots, I_{k}$ are ideals in $A$ which are contained in $Q$, then $C+I_{1}^{e_{1}}+\cdots+I_{k}^{e_{k}} M$ covers $B+I_{1}^{e_{1}}+\cdots+I_{k}^{e}$, for all integers $e_{i} \geqq 1$. Also, the $M$ primary components of $B$ and $B+I_{1}^{e_{1}}+\cdots+I_{k}^{e_{k}}$ are the same.

Proof. The first statement is clear by (2.18.2). The last state- 
ment follows from the facts: $Q$ contains $D=B+I_{1}^{e_{1}}+\cdots+I_{k}^{e}$; $D$ has an $M$-primary component (2.17); and, $D \supseteq B$.

If $B_{0} \subset B_{1} \subset \cdots \subset B_{n}$ is a composition series of $M$-primary ideals in a Noetherian ring $A$ and $I^{e} \subseteq B_{0}$, then it is clear that the images of the $B_{i}$ in $A / I^{e}$ is a composition series of $M / I^{e}$-primary ideals. The following result, which is closely related to (2.18.2) and (2.19), shows that if $I \cong M$ and we no longer assume that the $B_{i}$ are $M$-primary (so possibly $I^{e} \nsubseteq B_{0}$, for all $e \geqq 1$ ), then, even so, the images of the $B_{i}$ in $A / I^{e}$ are still a composition series.

Proposition 2.20. Let $B, C, I$, and $M$ be ideals in a Noetherian ring $A$ such that $M$ is maximal, $C M$-covers $B$, and $I \subseteq M$. Then $C+I^{e} M$-covers $B+I^{e}$, for all large integers $e$.

Proof. For all large $e, I^{e}$ is contained in an $M$-primary component of $B$ (2.17), so $C \cap I^{e}=B \cap I^{e}$, by (2.17). Therefore $C+I^{e}$ $M$-covers $B+I^{e}(2.13)$.

If $A$ is local, then an alternate proof of (2.20) which does not use (2.17) is: since, for each ideal $D$ in $A, D=\cap\left\{D+I^{e} ; e=1,2, \cdots\right\}$, $B_{e}=B+I^{e} \subset C+I^{e}=C_{e}$, for all large $e$, so $C_{e}$ covers $B_{e}$ (2.14.3).

Further results concerning ideal covers are given in (3.2), (3.9), (3.10), and (3.16.2).

3. Notes on associated primes and prime divisors. In this section, we apply the results in $\S 2$ to obtain some results on Bourbaki associated primes in arbitrary rings and prime divisors in Noetherian rings. (We prefer to use the prime divisor terminology in Noetherian rings, since it is somewhat more standard and the two concepts of associated primes are equivalent.) To begin, recall the following definition.

Definition 3.1. A prime ideal $P$ in a ring $A$ is a Bourbaki associated prime of an ideal $I$ in $A$ in case there exists $x \in A$ such that $I: x A=P$.

It is known $[6,(1.2)]$ that if $P$ is a Bourbaki associated prime of $I$, then $P$ is a prime divisor of $I$ in Nagata's terminology [5, p. 19]. (See also $[5,(8.8)]$.)

REMARK 3.2. Let $B$ and $M$ be ideals in a ring $A$ such that $M$ is maximal. Then $B$ is $M$-covered if and only if $M$ is a Bourbaki associated prime of $B$, by (2.2.3).

Because of (3.2), a number of results in $\S 2$ concerning an $M$ - 
covered ideal $I$ can be translated to results concerning a Bourbaki associated prime of $I$. (3.3)-(3.6) correspond to (2.3) and some of its corollaries.

Corollary 3.3. Let $I$ and $M$ be ideals in a ring $A$ such that $M$ is maximal and $M I \neq I$. Then $M I$ is an intersection of ideals $J \subset I$ such that $I$ lies immediately above $J$ and $J$ has $M$ as a Bourbaki associated prime.

Proof. By (2.3), $M I$ is an intersection of ideals $J M$-covered by $I$. Therefore, for each such $J, I$ lies immediately above $J$, and $M$ is a Bourbaki associated prime of $J$ (3.2).

CoRollary 3.4. Let $I \neq(0)$ and $M$ be ideals in a ring $A$ such that $I$ is finitely generated and $M$ is maximal. Then $I$ lies immediately above an ideal $J$ that has $M$ as a Bourbaki associated prime if and only if (0): $I \subseteq M$, and then $M I$ is an intersection of such ideals. (Thus, if $A$ is either quasi-local or an integral domain, then $M I$ is an intersection of such ideals.)

Proof. This is clear by (2.4), (2.5), and (3.2).

A particularly important case of (3.4) is when $A$ is Noetherian.

Corollary 3.5. For each ring $A$ and for each ideal $D \neq A, D$ is an intersection of ideals $J$ each having a maximal ideal as a Bourbaki associated prime. Further, every nonzero ideal I contains an ideal with a maximal ideal as a Bourbaki associated prime.

Proof. This follows immediately from (2.6) and (3.2).

(3.5) generalizes the well-known result that if $p \subset P$ are prime ideals in a Noetherian ring $A$ such that $p A_{P} \neq(0)$, then $P$ is an imbedded prime divisor of some ideal contained in $p$. Namely, by the last statement in (3.5), $P A_{P}$ is a prime divisor of some ideal, say $B A_{P}$, contained in $p A_{P}$, so, since $A$ is Noetherian, $P$ is a prime divisor of $B$ and $B \subset p$.

(3.6) stands in relation to (3.5) in the same way that (2.7) stands to $(2.6)$.

CoROLLARY 3.6. Let $I \subseteq M$ be ideals in a ring $A$ such that $M$ is maximal. Then $I$ is an intersection of ideals each of which has $M$ as a Bourbaki associated prime in the following two cases:

(3.6.1) I is prime. 
(3.6.2) $M$ is finitely generated and $I=I A_{M} \cap A$.

Proof. This is clear by (2.7) and (3.2).

The following result is a corollary to (2.11). The reason the converse of the corollary does not hold (as it did in (2.11)) is that, in general, an $M$-covered ideal has more than one $M$-cover. This follows, for example, from the result of Gröbner mentioned in the introduction.

Corollary 3.7. Let $D$ and $M$ be ideals in a ring $A$ such that $M$ is maximal, and let $x \in A$. If $D: x A \subseteq M$, then $M$ is a Bourbaki associated prime of $D+x M$.

Proof. If $D: x A \subseteq M$, then $D+x A M$-covers $D+x M$, by (2.11), so $M$ is a Bourbaki associated prime of $D+x M$ (3.2).

(3.8) corresponds to (2.12).

REMARK 3.8. Let $D$ and $M$ be ideals in a ring $A$ such that $M$ is maximal. Then the following statements hold:

(3.8.1) If $D \subseteq M$, then $M$ is a Bourbaki associated prime of $D+x M$ for all $x \in A$, $\notin M$.

(3.8.2) If $A$ is quasi-local and $M$ is a Bourbaki associated prime of $D$, then, for all finitely generated ideals $I$ in $A, M$ is a Bourbaki associated prime of $D+I M$.

Proof. (3.8.1) follows from (2.12.1) and (3.2), and (3.8.2) follows from (2.12.2) and (3.2).

The next corollary to (3.2) gives another application of the covering relationship.

CoRollary 3.9. Let $b_{1}, \cdots, b_{k}$ be an $R$-sequence in a local ring $(R, M) . \quad B=\left(b_{1}, \cdots, b_{k}\right) R$ is covered if and only if every maximal $R$-sequence has length equal to $k$.

Proof. If $B$ is covered, then $M$ is a prime divisor of $B$ (3.2), so $b_{1}, \cdots, b_{l c}$ is a maximal $R$-sequence, hence every maximal $R$ sequence has length equal to $k$ [10, Theorem 1, p. 396].

If every maximal $R$-sequence has length equal to $k$, then $M$ is a prime divisor of $B$, so $B$ is covered (3.2).

A result related to (3.9) is given in (3.19). 
The next result is another corollary to (3.2). However, it is of sufficient importance to be called a theorem. It characterizes finitely generated Bourbaki associated primes in an arbitrary ring in terms of ideal covers.

THEOREM 3.10. Let $B$ and $P$ be ideals in a ring $A$ such that $P$ is a finitely generated prime ideal. Then $P$ is a Bourbaki associated prime of $B$ if and only if $B A_{P}$ is covered.

Proof. Since $P$ is finitely generated, $P$ is a Bourbaki associated prime of $B$ if and only if $P A_{P}$ is a Bourbaki associated prime of $B A_{P}$ [1, Proposition 5, p. 134] if and only if $B A_{P}$ is covered (3.2).

A number of corollaries of (3.2) and (3.10) will now be given. In these corollaries, results in $\S 2$ concerning a covered ideal $B$ in an arbitrary ring correspond to results concerning finitely generated Bourbaki associated primes of $B$. (3.11) corresponds to (2.13), (2.14.1), and (2.14.2). (3.11) is of some interest, since if $B \subset D \subset P$ are ideals in a Noetherian ring $A$ such that $P$ is an imbedded prime divisor of $B$, then it need not be true that $P$ is a prime divisor of $D$. However, (3.11) shows that, for each ideal $I$ such that $P$ is not a prime divisor of $B \cap I, P$ is a prime divisor of $B+J$, for all ideals $J \subseteq I . \quad$ ((3.8.2) showed a closely related result.) (3.11) also shows that for all ideals $I \nsubseteq P, P$ is a prime divisor of $B \cap I$.

Corollary 3.11. Let $B, I$, and $P$ be ideals in a ring $A$ such that $P$ is a Bourbaki associated prime of $B$ and is either finitely generated or maximal. Then $P$ is a Bourbaki associated prime of either $B+J$, for all ideals $J \subseteq I$, or $B \cap K$, for all ideals $K \supseteqq I$.

Proof. Assume $P$ is finitely generated, let $C$ be an ideal in $A$ such that $C A_{P}$ covers $B A_{P}$ (3.10), and assume that there exists an ideal $J \subseteq I$ such that $P$ is not a Bourbaki associated prime of $B+J$. Then $(B+J) A_{P}$ is not covered (3.10), so $(C+J) A_{P}$ does not cover $(B+J) A_{P}$, hence $(C+I) A_{P}$ does not cover $(B+I) A_{P}$ (2.14.1). Therefore $(C \cap I) A_{P}$ covers $(B \cap I) A_{P}(2.13)$, so, by (2.14.2), $(C \cap K) A_{P}$ covers $(B \cap K) A_{P}$, for all ideals $K \supseteqq I$. Therefore the conclusion follows from (3.10). The proof is similar using (3.2) and avoiding localization, if $P$ is maximal.

(3.12) corresponds to (2.15).

Corollary 3.12. Let $B, I$, and $P$ be as in (3.11). Then the following statements hold: 
(3.12.1) If $P$ is not a Bourbaki associated prime of $B \cap I^{e}$, for some $e \geqq 1$, then $P$ is a Bourbaki associated prime of $B+I^{i}$, for all $i \geqq e$.

(3.12.2) If $P$ is not a Bourbaki associated prime of $B+I^{e}$, for some $e \geqq 1$, then $P$ is a Bourbaki associated prime of $B \cap I^{i}$, for $i=1, \cdots, e$.

(3.12.3) If $P$ is not a Bourbaki associated prime of $B+d^{e} A$, for some $e \geqq 1$, and if $d$ is regular, then $P$ is a Bourbaki associated prime of $B: d^{i} A$, for $i=1, \cdots, e$.

Proof. (3.12.1) and (3.12.2) are clear by (3.11).

(3.12.3) Assume that $P$ is finitely generated, let $C$ be an ideal in $A$ such that $C A_{P}$ covers $B A_{P}(3.10)$, and assume that $P$ is not a Bourbaki associated prime of $B+d^{e} A$. Then $\left(C+d^{e} A\right) A_{P}$ does not cover $\left(B+d^{e} A\right) A_{P} \quad(3.10)$, so $\left(C+d^{e} A\right) A_{P}=\left(B+d^{e} A\right) A_{P}$ (2.14.3), hence, by $(2.15 .3),\left(C: d^{i} A\right) A_{P}$ covers $\left(B: d^{i} A\right) A_{P}$, for $i=1, \cdots, e$. Therefore the conclusion follows from (3.10). If $P$ is maximal, the proof is similar using (3.2) and avoiding localization.

It is clear that the contrapositive of (3.12.3) shows that if $P$ is not a Bourbaki associated prime of $B: d^{e} A$, for some $e \geqq 1$, then $P$ is a Bourbaki associated prime of $B+d^{i} A$, for all $i \geqq e$.

The next corollary corresponds to (2.16).

Corollary 3.13. Let $I$ and $P$ be ideals in a ring $A$ such that $P$ is a Bourbaki associated prime of $d I$ and is either maximal or finitely generated, where $d$ is a regular element in $A$. Then $P$ is a Bourbaki associated prime of either $d A$ or $I$. (Hence, if $d \notin P$, then $P$ is a Bourbaki associated prime of $I$.)

Proof. If $P$ is finitely generated, then $d I A_{P}$ is covered (3.10), so either $d A_{P}$ or $I A_{P}$ is covered (2.16). Therefore the conclusion follows from (3.10). The proof is similar using (3.2) and avoiding localization, if $P$ is maximal.

(3.13) shows that if $P$ is a Bourbaki associated prime of $d^{e} I$, for some $e \geqq 1$, then $P$ is a Bourbaki associated prime of either $d A$ or $d^{i} I$, for $i=0,1, \cdots, e$. Namely, if $P$ is not a Bourbaki associated prime of $d A$, then $P$ is a Bourbaki associated prime of $d^{e-1} I$ (since $\left.d^{e} I=d\left(d^{e-1} I\right)\right)$, so the conclusion follows by repetition.

Also, if $A$ is a Krull domain, if $P$ is either a finitely generated prime ideal or a maximal ideal in $A$, and if $P$ is a Bourbaki associated prime of $d q(d \in A$ and $q$ a primary ideal in $A)$ and height $P>1$, then $q$ is $P$-primary, by (3.13) (since nonzero principal ideals in a 
Krull domain are finite intersections of height one primary ideals [5, (33.3)]). In terms of ideal covers, this says: if $d q$ is $P$-covered ( $P$ maximal and height $P>1$ ), then $q$ is primary (2.16).

REMARK 3.14. (3.14.1) (3.10) and its corollaries hold for all ideals $B$ and $P$ ( $P$ prime), if $A$ is Noetherian.

(3.14.2) It is known [1, Chapter 4, Ex. 1c, p. 163] that if $P$ in (3.10) is not finitely generated, then there may exist an ideal $B \subset P$ such that $P A_{P}$ is a Bourbaki associated prime of $B A_{P}$ (so $B A_{P}$ is covered) and $P$ is not a Bourbaki associated prime of $B$.

The following result is a corollary to (3.10), (2.19), and (2.20), but, due to its importance, we call it a theorem. The theorem corresponds to (2.19) and (2.20).

THEOREM 3.15. Let $P$ be a prime divisor of an ideal $B$ in a Noetherian ring $A$, let $Q$ be a P-primary component of $B$, and let $I_{1}, \cdots, I_{k}$ be ideals contained in $P$. Then the following statements hold:

(3.15.1) If $I_{1}, \cdots, I_{k} \subseteq Q$, then $P$ is a prime divisor of $B+I_{1}^{e_{1}}+\cdots+I_{k}^{e_{k}}$, for all integers $e_{i} \geqq 1$.

(3.15.2) For all large integers $e_{i}, P$ is a prime divisor of $B+I_{1}^{e_{1}}+\cdots+I_{k}^{e}$.

(3.15.3) In both cases the P-primary component of $B+I_{1}^{e_{1}}+$ $\cdots+I_{k}^{e_{k}}$ is $Q$.

Proof. (3.15.1) It clearly suffices to prove if $I \leqq Q$, then $P$ is a prime divisor of $B+I$. For this, $B A_{P}$ is covered (3.10), so $(B+I) A_{P}$ is covered (2.18.2), hence $P$ is a prime divisor of $B+I$ (3.10).

(3.15.2) follows similarly from (2.20), and (3.15.3) follows from (2.19).

This section will be closed with a number of corollaries to (3.15). For the first corollary, recall that if $P$ is a prime divisor of $d A$ in a Noetherian ring $A$ and $d$ is regular, then $P$ is a prime divisor of $c A$, for all regular elements $c \in P[5,(12.6)]$. (3.16) shows that a somewhat analogous result holds if $P$ is a prime divisor of zero.

Corollary 3.16. Assume that $P$ is a prime divisor of (0) in a ring $A$. Then the following statements hold:

(3.16.1) If $A$ is Noetherian, then, for all ideals $I_{1}, \cdots, I_{k} \subseteq P$, $P$ is a prime divisor of $I_{1}^{e_{1}}+\cdots+I_{k}^{e}$, for all large $e_{i}$.

(3.16.2) If $(A, P)$ is quasi-local, then, for each $d \in P$ such that (0): $P \nsubseteq d A, d A$ is covered (so $P$ is a Bourbaki associated prime of $d A)$. 


\section{Proof. (3.16.1) is clear by (3.15).}

(3.16.2) Assume that $(A, P)$ is quasi-local and let $d \in P$ such that (0): $P \nsubseteq d A$. Let $c \in(0): P$, $\notin d A$. Then $P \subseteq(0): c A \subseteq d A: c A \neq A$, so $P$ is a Bourbaki associated prime of $d A$, hence $d A$ is covered (3.2).

The following result shows that, for certain prime ideals $p$ and for all large $e, p^{e} \neq p^{(e)}$, the $e$ th symbolic power of $p$.

COROLlaRy 3.17. If $P$ is a prime divisor of (0) in a Noetherian ring $A$, then for all prime ideals $p \subset P$ and for all large $e, p^{e}$ is not p-primary.

Proof. By (3.16.1), $P$ is a prime divisor of $p^{e}$, for all large $e$.

The hypothesis in the next corollary is somewhat restrictive, but an example following the result shows that the conclusion does not hold for all local rings. The corollary shows, in particular, that if $P$ is a prime divisor of an ideal $B$ which is generated by $k$ elements, if height $B=n$, and if height $P=h$, then $P$ is an prime divisor of an ideal $C_{i}$ generated by $k+i$ elements such that height $C_{i}=n+i$, for $i=1, \cdots, h-n$. In particular, if $(A, M)$ is a catenary local domain of altitude $h$, and if $M$ is a prime divisor of a principal ideal, then $M$ is a prime divisor of an ideal of height $i$ and generated by $i$ elements, for $i=1, \cdots, h$.

CoROLlaRY 3.18. Let $A$ be a local ring that satisfies the first chain condition for prime ideals, let $B$ be an ideal in $A$, and let $P$ be a prime divisor of $B$. Let height $P=h$ and let height $B=n$. Then, for $i=1, \cdots, h-n, P$ is a prime divisor of an ideal $C_{i}=$ $\left(B, d_{1}, \cdots, d_{i}\right) A$ such that height $C_{i}=n+i$.

Proof. It may clearly be assumed that $h>n$, and it clearly suffices to prove that $P$ is a prime divisor of an ideal $C=B+d A$ such that height $C=n+1$. For this, let $x \in P$ such that $x$ is not in any prime divisor of $B$ that has height $=n$. Then height $B+$ $x A \geqq n+1$. Now, if $p$ is a height $n$ prime divisor of $B$, then height $p+x A=n+1$, by the first chain condition (since $p+x A \neq A$ ), and $B+x A \subseteq p+x A$, so it follows that height $B+x A=n+1$. Therefore, let $d=x^{e}$ with $e$ large, so $P$ is a prime divisor of $B+d A$ (3.15) and height $B+d A=n+1$.

The conclusion of (3.18) does not hold for arbitrary local rings. For example, let $(A, P)$ be a local ring such that altitude $A=a>1$ 
and there exists a depth one minimal prime ideal $z$ in $A$. Let $Q$ be a $P$-primary ideal such that $z \nsubseteq Q$, and let $B=z \cap Q$. Then height $B=0$ and $P$ is a prime divisor of $B$, but for all $d \in M$ such that height $B+d A>0$, height $B+d A=a>1$.

Corollary 3.19. Let $(R, M)$ be a local ring, let $b_{1}, \cdots, b_{k}$ be an $R$-sequence, and assume that $M$ is a prime divisor of $\left(b_{1}, \cdots, b_{k}\right) R$. Then, for all $R$-sequences $c_{1}, \cdots, c_{k}$ and all elements $d_{1}, \cdots, d_{h}(h \geqq 0)$ in $M, M$ is a prime divisor of $\left(c_{1}^{f_{1}}, \cdots, c_{k}^{f_{k}}, d_{1}^{e_{1}}, \cdots, d_{h}^{e_{h}}\right) R$, for all $f_{i} \geqq 1$ and for all large $e_{j}$.

Proof. $c_{1}^{f_{1}}, \cdots, c_{k}^{f_{k}}$ is an $R$-sequence [3, Exercise 12, p. 102], so $M$ is a prime divisor of $\left(c_{1}^{f_{1}}, \cdots, c_{k}^{f_{k}}\right) R[10$, Theorem 1, p. 396]. Therefore the conclusion follows from (3.15.2).

4. Modules and $M$-covers. In this section, we briefly indicate how the results in $\S 2$ can be generalized to $A$-modules. We begin by recalling the definition.

Definition 4.1. Let $A$ be a ring, let $M$ be a maximal ideal in $A$, and let $\mathscr{B}$ and $\mathscr{C}$ be submodules of an $A$-module $\mathscr{M}$. Then $\mathscr{C}$ $M$-covers $\mathscr{B}$ in case $\mathscr{B} \subset \mathscr{C}$ and $\mathscr{C} / \mathscr{B} \cong A / M$. $\mathscr{C}$ covers $\mathscr{B}$ in case $\mathscr{C} N$-covers $\mathscr{B}$, for some maximal ideal $N$ in $A$, and $\mathscr{B}$ is covered in case some submodule of $\mathscr{L}$ covers $\mathscr{B}$.

Given a ring $A$ and an $A$-module $\mathscr{A}$, recall that if $\mathscr{B}$ is a submodule of $\mathscr{C}$, then (0) $\oplus \mathscr{B}$ is an ideal in the ring $A \oplus \mathscr{C}$, where addition is componentwise and multiplication is given by

$$
(\alpha, m)\left(a^{\prime}, m^{\prime}\right)=\left(\alpha \alpha^{\prime}, \alpha m^{\prime}+\alpha^{\prime} m\right)
$$

(the principle of idealization $[5, \mathrm{p} .2]$ ).

REMARK 4.2. With the notation of (4.1), $\mathscr{C} M$-covers $\mathscr{B}$ if and only if $(0) \oplus \mathscr{C}(M \oplus \mathscr{C})$-covers $(0) \oplus \mathscr{B}$ in $A \oplus \mathscr{M}$.

Proof. This follows immediately from

$$
\mathscr{C} \mid \mathscr{B} \cong((0) \oplus \mathscr{C}) /((0) \oplus \mathscr{B}) \text {. }
$$

Using (4.2), it is straightforward to generalize most of the results of $\S 2$ to submodules of an arbitrary $A$-module $\mathscr{M}$. In particular, the generalizations (both statements and proofs) of (2.2), (2.3), (2.6), (2.7.2), (2.8), the first statement in (2.9), (2.11), (2.13), and (2.14.1)-(2.14.3) are clear; and, for (2.14.6), consider maximal submodules of $\mathscr{M}$.

The integral domain parts of (2.4) and (2.10) do not generalize 
by this method, since $A \oplus \mathscr{C}$ is a domain only if $\mathscr{C}=(0)$. The quasi-local parts of (2.9), (2.10), and (2.12.2) do generalize, since $A \oplus \mathscr{C l}$ is quasi-local if $A$ is. This also holds for the quasi-local part of (2.4), once it is shown that the main part of (2.4) generalizes (see below), and then (2.5) also generalizes.

(2.7.1) and (2.14.5) do not generalize in a convenient manner by this method.

The generalizations of the remaining results in $\S 2$ are somewhat less clear, so they will now be explicitly stated. In the statements, $A$ is a ring, $\mathscr{C}$ is an $A$-module, and $M$ is a maximal ideal in $A$. Script letters will denote submodules of $\mathscr{A}$.

(4.4) $\mathscr{F} M$-covers some $\mathscr{J}$ if and only if $(0): \mathscr{I} \subseteq M$.

Proof. $\mathscr{I} M$-covers some $\mathscr{J}$ if and only if $(0) \oplus \mathscr{I} M \oplus \mathscr{M}$ covers some $(0) \oplus \mathscr{J}$, by (4.2), if and only if $((0) \oplus(0)):((0) \oplus \mathscr{I}) \subseteq$ $M \oplus . \mathscr{l}$, by (2.4): that is, if and only if $\left(\left(\left(0_{A}\right):\left(0_{A}\right)\right) \cap\left(0_{\mathscr{A}}\right): \mathscr{F}\right) \oplus$ $\left(\left(0_{\mathscr{M}}\right):\left(0_{A}\right)\right) \subseteq M \oplus \mathscr{C}$, and this holds if and only if $\left(0_{\mathscr{M}}\right): \mathscr{I} \subseteq M$.

(4.12.1) If $\mathscr{D} \subseteq M \mathscr{l}$, then, for all $x \in \mathscr{L}, \notin M \mathscr{L}, \mathscr{D}+(x)$ $M$-covers $\mathscr{D}+M x$.

Proof. If $\mathscr{D} \subseteq M \mathscr{C l}$ and $x \notin M \mathscr{L}$, then $((0) \oplus \mathscr{D}):(0, x)(A \oplus \mathscr{C})=(\mathscr{D}:(x)) \oplus \mathscr{M} \leqq M \oplus \mathscr{C}$,

so $(0) \oplus(\mathscr{D}+(x)) \quad(M \oplus \mathscr{C})$-covers $(0) \oplus(\mathscr{D}+M x)$ (2.11), hence $\mathscr{D}+(x) M$-covers $\mathscr{D}+M x(4.2)$.

(4.14.4) Assume that $\mathscr{C} M$-covers $\mathscr{B}$. Then the following statements hold:

(a) For each $\mathscr{C}$-regular element $d \in A$, either $\mathscr{C}: d A=\mathscr{B}: d A$ or $\mathscr{C}: d A M$-covers $\mathscr{B}: d A$.

(b) For each regular element $x \in \mathscr{C}$ (that is, if $a \in A$ and $a x=0$, then $a=0)$, either $\mathscr{C}:(x)=\mathscr{B}:(x)$ or $\mathscr{C}:(x) M$-covers $\mathscr{B}:(x)$.

Proof. By (2.14.3) (for modules), either $\mathscr{C} \cap d \mathscr{M}=\mathscr{B} \cap d \mathscr{M}$ or $\mathscr{C} \cap d \mathscr{C} \quad M$-covers $\mathscr{B} \cap d \mathscr{A}$. Therefore, since $d(\mathscr{D}: d A)=$ $\mathscr{D} \cap \mathrm{d} \mathscr{C}$ and $d$ is $\mathscr{C}$-regular, (a) follows.

The proof of (b) is similar.

(4.15.1) Assume that $\mathscr{C} M$-covers $\mathscr{B}$, and let $I$ be an ideal in $A$. If $\mathscr{C} \cap I^{e} \mathscr{M}=\mathscr{B} \cap I^{e} \mathscr{M}$, for some $e \geqq 1$, then $\mathscr{C}+I^{i} \mathscr{M} M$ covers $\mathscr{B}+I^{i} \mathscr{A}$, for all $i \geqq e$. 
The proof is similar to the proof of (2.15.1).

(2.15.2) generalizes in a similar manner.

Two generalizations of (2.15.3) similar to the two generalizations of (2.14.4) given above can now be easily supplied by the reader. (For the case $x \in \mathscr{C l}$, the exponent $e$ is, of course, $e=1$.)

(4.16) Let $\mathscr{J}$ be a submodule of $\mathscr{L}$, and let $d$ be $\mathscr{C}$-regular in $A$. If $d \mathscr{J}$ is $M$-covered, then either $d \mathscr{M}$ or $\mathscr{J}$ is $M$-covered.

The proof is similar to the proof of (2.16).

The following result follows quite readily from (2.17), by using the principle of idealization. However, some of the specific results used in its proof are, perhaps, not well-known. For this reason, we give a fairly detailed proof of the result.

(4.17) Let $A$ be a Noetherian ring, let $\mathscr{C}$ be a finitely generated $A$-module, let $M$ be a maximal ideal in $A$, and let $\mathscr{B} \subset \mathscr{C}$ be submodules of $\mathscr{C}$ such that $\mathscr{C} M$-covers $\mathscr{B}$. Then $\mathscr{B}$ has an $M$ primary component, $\mathscr{B}$ and $\mathscr{C}$ have the same primary decompositions except for $M$-primary components, and either some $M$-primary component of $\mathscr{C} M$-covers some $M$-primary component of $\mathscr{B}$ or $\mathscr{C}$ has no $M$-primary component.

Proof. $R=A \oplus \mathscr{C}$ is Noetherian and $C=(0) \oplus \mathscr{C} M \oplus \mathscr{K}$ covers $(0) \oplus \mathscr{B}=B$, so by (2.17) and its proof, there exist ideals $I$ and $q$ in $R$ such that $q$ is $M \oplus \mathscr{M}$-primary, $I:(M \oplus \mathscr{M})=I$, $B=I \cap q$, and either $C=I$ or $M \oplus \mathscr{C}$ is a prime divisor of $C$ and $C=I \cap q^{\prime}$, and it may then be assumed that $q^{\prime} M \oplus \mathscr{C}$-covers $q$. Now, since $q$ is $M \oplus \mathscr{C}$-primary, either $q=Q \oplus \mathscr{C}$ or $Q \oplus \mathscr{N}$, for some $M$-primary ideal $Q$ and for some $M$-primary submodule $\mathscr{N}$ of $\mathscr{C}$ (see [8, Lemma 1.26], where it is shown that $q$ must be of this form; see [9, pp. 252-253] for some results on primary submodules). Also, if $M \oplus \mathscr{C}$ is a prime divisor of $C$, then $q^{\prime}=Q^{\prime} \oplus \mathscr{N}^{\prime}$ with $Q^{\prime} M$-primary and either $\mathscr{N}^{\prime}$ an $M$-primary submodule of $\mathscr{C}$ or $\mathscr{N}^{\prime}=\mathscr{A}$. Moreover, $I=J \oplus \mathscr{F}$, for some ideal $J$ in $A$ and for some submodule $\mathscr{I}$ of $\mathscr{K}$.

Since $C$ covers $B$, since the first components of $B$ and $C$ are (0), and since the second component of $B=I \cap q$ is either $\mathscr{J}$ or $\mathscr{F} \cap \mathscr{N}$ and the second component of $C$ is either $\mathscr{I}$ or $\mathscr{I} \cap \mathscr{N}^{\prime}$, it follows that $B=(0) \oplus(\mathscr{J} \cap \mathscr{N})$ and $q=Q \oplus \mathscr{N}$. Therefore, $\mathscr{B}=\mathscr{J} \cap \mathscr{N}$, so if $M \oplus \mathscr{C}$ is not a prime divisor of $C$, then $(0) \oplus \mathscr{C}=C=I=J \oplus \mathscr{F}$, so $\mathscr{C}=\mathscr{F}$, and the conclusion holds (since $I:(M \oplus \mathscr{C})=I$ implies $\mathscr{I}: M=\mathscr{J})$. Therefore assume that $M \oplus \mathscr{C}$ is a prime divisor of $C$, so $C=I \cap q^{\prime}=\left(J \cap Q^{\prime}\right) \oplus\left(\mathscr{I} \cap \mathscr{N}^{\prime}\right) \quad\left(\right.$ possibly $\left.\mathscr{N}^{\prime}=\mathscr{M}\right)$. Therefore, since $C$ covers $B$ and the first components are (0), $\mathscr{I} \cap \mathscr{N}^{\prime}$ covers $\mathscr{I} \cap \mathscr{N}$, so it may be assumed that $\mathscr{N}^{\prime} \supset \mathscr{N} \nsupseteq \mathscr{F}$. Thus since $q=Q \oplus \mathscr{N}$ is covered by $q^{\prime}$, it follows that $q^{\prime}=Q \oplus \mathscr{N}^{\prime}$ 
(same $Q$ as for $q$ ) and $\mathscr{N}^{\prime}$ covers $\mathscr{N}$ (possibly $\mathscr{N}^{\prime}=\mathscr{K}$ ). Finally, it has already been shown that $\mathscr{B}=\mathscr{I} \cap \mathscr{N}, \mathscr{C}=\mathscr{I} \cap \mathscr{N}^{\prime}$, and $\mathscr{I}: M=\mathscr{F}$.

(4.18) Assume that $A$ is Noetherian, that $\mathscr{M}$ is finitely generated, and that $\mathscr{C} M$-covers $\mathscr{B}$, and let $J$ be an ideal in $A$.

(4.18.1) If $J \subseteq M=\mathscr{B}: \mathscr{C}$, then $\mathscr{C}: J^{i} \mathscr{M}=\mathscr{B}: J^{i} \mathscr{M}$ and $\mathscr{C}: J^{i}=\mathscr{B}: J^{i}$, for all large $i$.

(4.18.2) If $\mathscr{N}$ is an $M$-primary component of $\mathscr{B}$ and $J \mathscr{M} \subseteq \mathscr{N}$; then $\mathscr{C}+J^{i} \mathscr{M} M$-covers $B+J^{i} \mathscr{M}$, for all $i \geqq 1$.

Proof. (4.18.1) By [9, p. 252], and with the notation of (4.17), $M^{i} \mathscr{L} \subseteq \mathscr{N}$, for all large $i$, so $\mathscr{B}: J^{i} \mathscr{M}=\mathscr{C}: J^{i} \mathscr{M}$. Also, $\mathscr{K}=$ $\mathscr{N}: J^{i}=\mathscr{N}^{\prime}: J^{i}$, so $\mathscr{B}: J^{i}=\mathscr{J}: J^{i}=\mathscr{C}: J^{i}$, for all large $i$.

The proof of (4.18.2) is similar to that of (2.18.2).

(2.19) can be generalized in the same way that (4.18) generalizes (2.18).

(4.20) Let $A, \mathscr{B}, \mathscr{C}, M$, and $J$ be as in (4.18), and assume that $J \subseteq M$. Then $\mathscr{C}+J^{e} \mathscr{M} M$-covers $\mathscr{B}+J^{e} \mathscr{M}$, for all large $e$.

The proof is similar to the proof of $(2.20)$, using [9, p. 252].

5. Modules and Bourbaki associated primes. In this section, we briefly indicate how the results in $\S 3$ can be generalized to $A$ modules. We begin by recalling the following definition.

Definition 5.1. Let $A$ be a ring and $\mathscr{C}$ an $A$-module. Then a prime ideal $P$ in $A$ is a Bourbaki associated prime of a submodule $\mathscr{B}$ of $\mathscr{C}$ in case there exists $x \in \mathscr{M}$ such that $P=\mathscr{B}:(x)$.

The following remark generalizes (3.2).

REMARK 5.2. Let $\mathscr{B}$ be a submodule of an $A$-module $\mathscr{A}$, and let $M$ be a maximal ideal in $A$. Then $\mathscr{B}$ is $M$-covered if and only if $M$ is a Bourbaki associated prime of $\mathscr{B}$.

Proof. This follows easily from (2.2.3) generalized to modules.

Using (5.2) and the results in $\S 4$, the following results can easily be generalized to an arbitrary $A$-module $\mathscr{L}:(3.3),(3.4),(3.5)$, (3.6.2), (3.7), (3.8), (3.9) (let $\mathscr{C}$ be a finitely generated $R$-module and $b_{1}, \cdots, b_{k}$ an $\mathscr{C}$-sequence), (3.10), (3.11), (3.12.1) and (3.12.2) (let $I$ be an ideal as in (4.15.1) and the generalization of (2.15.2)), (3.12.3) (this has the same two generalizations that (2.15.3) had in §4), (3.13) (let $\mathscr{F}$ and $d$ be as in (4.16); the parenthetical statement does not 
generalize in a convenient manner), (3.14.1) (if $A$ is Noetherian, then the results hold for all prime ideals and for all $A$-modules), (3.14.2) (this shows the results do not hold for arbitrary modules over an arbitrary ring), (3.15) (assume that $A$ is Noetherian, that $\mathscr{C}$ is finitely generated, and that $I_{1}, \cdots, I_{k}$ are ideals contained in $P$ as in the generalization of (2.19) in $\S 4),(3.16 .1)$ (same assumptions as for the generalization of (3.15) and let $P$ be a Bourbaki associated prime of $(0)$ in $\mathscr{C}$ ), (3.16.2) (let $P$ be a Bourbaki associated prime of $(0)$ in $\mathscr{C l}$ and let $x \in \mathscr{C}, \notin\left(0_{\mathscr{M}}\right): P$ ), and (3.19) (use the same assumptions as for the generalization of (3.9) and assume that $M$ is a Bourbaki associated prime of $\left.\left(b_{1}, \cdots, b_{k}\right) \mathscr{M l}\right)$.

(3.6.1), (3.17), and (3.18) do not generalize nicely by this method.

6. Sheltered modules. In this brief section, we consider a concept which is somewhat more restrictive than the covering relationship, namely, the sheltering relationship. We begin by recalling the definition.

Definition 6.1. [1, Chapter 3, Ex. 18, p. 110]. Let $\mathscr{t h}$ be an $A$-module, and let $\mathscr{B}$ be a submodule of $\mathscr{A}$. Then $\mathscr{B}$ is said to be sheltered in case the set of submodules of $\mathscr{C}$ which properly contain $\mathscr{B}$ contains a smallest element, which is then called the shelter of $\mathscr{B} . \mathscr{C}$ is sheltered in case $(0)$ is a sheltered submodule of $\mathscr{L}$.

The final result in this paper gives a few of the relationships between covered modules and sheltered modules.

REMARK 6.2. Let $A, \mathscr{B}$, and $\mathscr{C}$ be as in (6.1). Then the following statements hold:

(6.2.1) If $\mathscr{B}$ is sheltered, then $\mathscr{B}$ is $M$-covered, for some maximal ideal $M$ in $A$. Moreover, $M$ is uniquely determined, $M=$ $\mathscr{B}: \mathscr{C}$, where $\mathscr{C}$ is the shelter of $\mathscr{B}$, and then $\mathscr{C}$ is the unique cover of $\mathscr{B}$.

(6.2.2) [1, Chapter 3, Ex. 18a, p. 110]. $\mathscr{B}$ is the intersection of sheltered submodules of $\mathscr{M}$.

(6.2.3) (cf. (2.6).) Every proper submodule of $\mathscr{C}$ is an intersection of covered submodules. (In particular, every proper ideal in $A$ is an intersection of covered ideals.) Moreover, every nonzero submodule of $\mathscr{L}$ contains a covered submodule.

(6.2.4) If $(A, M)$ is a local ring and altitude $A>0$, then every nonzero ideal contains an $M$-covered ideal, but there exist nonzero ideals which do not contain a sheltered ideal.

(6.2.5) If $(A, M)$ is quasi-local and $\mathscr{B}$ has a unique $M$-cover, then $\mathscr{B}$ need not be sheltered. 
Proof. (6.2.1) $\mathscr{C} / \mathscr{B}$ is a nonzero simple $A$-module, so the conclusion follows from (2.2.1) (generalized to modules) and (4.21). (An alternate proof follows easily from [7, Theorem 6].)

(6.2.2) is given in the cited reference.

(6.2.3) The first statement follows from (6.2.1) and (6.2.2), and the parenthetical statement follows from this. Also, the last statement follows from (2.6) and (4.2).

(6.2.4) Assume that $(A, M)$ is a local ring and altitude $A>0$. Then every nonzero ideal contains an $M$-covered ideal (2.6). However, a sheltered ideal is $M$-primary [7, Theorem 6], so there exist nonzero ideals which do not contain a sheltered ideal.

(6.2.5) This is more or less clear, but a specific example will be given. Let $(R, N)$ be a local domain which is not a field, and let $A=R \oplus(R / N)$ (principle of idealization), so $A$ is a local ring with maximal ideal $M=N \oplus(R / N)$. Also, the zero ideal in $A$ has a unique $M$-cover (namely, $(0) \oplus(R / N)$ ). However, $A$ is not Artinian, since altitude $A=$ altitude $R>0$, so $A$ is not sheltered [7, Theorem $6]$.

\section{REFERENCES}

1. N. Bourbaki, Algebre Commutative, Chaps. 3 and 4, Actualités Scientifiques et Industrielles, no. 1293, Hermann, Paris, France, 1961.

2. W. Gröbner, Über irreduzible Ideale in kommutativen Ringen, Math. Ann. 110 (1935), 197-222.

3. I. Kaplansky, Commutative Rings, Allyn and Bacon, Boston, Mass., 1970.

4. R. Lee, Covers and associated primes in Noetherian lattice modules, Ph. D. Dissertation, University of California, Riverside, 1976.

5. M. Nagata, Local Rings, Interscience Tracts 13, Interscience Publishers, New York, N.Y., 1962.

6. D. H. Underwood, On some uniqueness question in primary representations of ideals, J. Math. Kyoto Univ., 9 (1969), 69-94.

7. S. Warner, Sheltered modules and rings, Proc. Amer. Math. Soc., 30 (1971), 8-14.

8. D. G. Whitman, Ring theoretic lattice modules and the Hilbert polynomial, Ph. D. Dissertation, Univ. Calif., Riverside, 1968.

9. O. Zariski and P. Samuel, Commutative Algebra, Vol. I, D. van Nostrand Co., Inc., New York, N.Y., 1958.

10. - Commutative Algebra, Vol. II, D. van Nostrand Co. Inc., New York, N.Y., 1960.

Received May 5, 1977 and in revised form July 20, 1977. The first author's research on this paper was supported in part by the National Science Foundation Grant NSF GP-28939-2.

UnIVERsity of CALIFornia

Riverside, CA 92521 



\section{PACIFIC JOURNAL OF MATHEMATICS}

EDITORS

RICHARD ARENS (Managing Editor)

University of California

Los Angeles, California 90024

C. W. Curtis

University of Oregon

Eugene, OR 97403

C. C. MOORE

J. DUGUNDJI

Department of Mathematics University of Southern Californa Los Angeles, California 90007

R. Finn AND J. Milgram Stanford University Stanford, California 94305

University of California

Berkeley, CA 94720

\section{ASSOCIATE EDITORS}

E. F. BeCKENBACH

B. H. NeUMANN

F. WOLF

K. YosHida

\section{SUPPORTING INSTITUTIONS}

UNIVERSITY OF BRITISH COLUMBIA UNIVERSITY OF SOUTHERN CALIFORNIA CALIFORNIA INSTITUTE OF TECHNOLOGY UNIVERSITY OF CALIFORNIA MONTANA STATE UNIVERSITY UNIVERSITY OF NEVADA, RENO STANFORD UNIVERSITY UNIVERSITY OF TOKYO UNIVERSITY OF UTAH NEW MEXICO STATE UNIVERSITY WASHINGTON STATE UNIVERSITY OREGON STATE UNIVERSITY UNIVERSITY OF WASHINGTON UNIVERSITY OF OREGON OSAKA UNIVERSITY 


\section{Pacific Journal of Mathematics \\ Vol. 73, No. 1 \\ March, 1977}

Thomas Robert Berger, Hall-Higman type theorems. $V \ldots \ldots \ldots \ldots \ldots \ldots \ldots$

Frank Peter Anthony Cass and Billy E. Rhoades, Mercerian theorems via

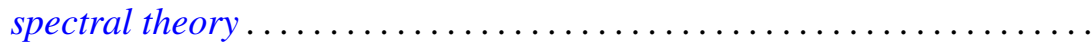

Morris Leroy Eaton and Michael David Perlman, Generating $\mathrm{O}(n)$ with

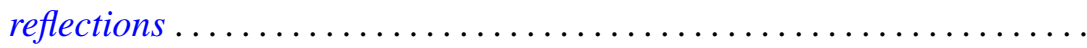

Frank John Forelli, Jr., A necessary condition on the extreme points of a

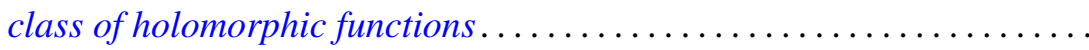

Melvin F. Janowitz, Complemented congruences on complemented

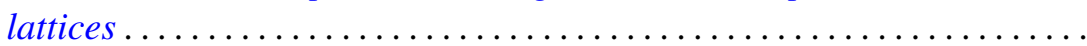

Maria M. Klawe, Semidirect product of semigroups in relation to amenability, cancellation properties, and strong $F \phi$ lner conditions....

Theodore Willis Laetsch, Normal cones, barrier cones, and the "spherical image" of convex surfaces in locally convex spaces ................

Chao-Chu Liang, Involutions fixing codimension two knots.............

Joyce Longman, On generalizations of alternative algebras .............

Giancarlo Mauceri, Square integrable representations and the Fourier

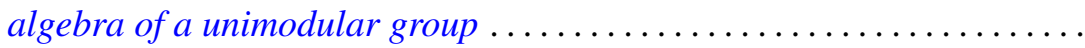

J. Marshall Osborn, Lie algebras with descending chain condition...

John Robert Quine, Jr., Tangent winding numbers and branched

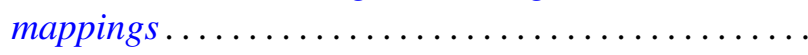

Louis Jackson Ratliff, Jr. and David Eugene Rush, Notes on ideal covers and associated primes .

H. B. Reiter and N. Stavrakas, On the compactness of the hyperspace of faces.

Walter Roth, A general Rudin-Carlson theorem in Banach-spaces ..

Mark Andrew Smith, Products of Banach spaces that are uniformly rotund in every direction.

Roger R. Smith, The R-Borel structure on a Choquet simplex ...

Gerald Stoller, The convergence-preserving rearrangements of real infinite series. ...

Graham H. Toomer, Generalized homotopy excision theorems modulo a Serre class of nilpotent groups...

Norris Freeman Weaver, Dehn's construction and the Poincaré conjecture....

Steven Howard Weintraub, Topological realization of equivariant intersection forms... 